\title{
Mortalidade prematura por doenças crônicas não transmissíveis e cobertura da atenção básica: análise dos indicadores
}

Premature mortality from chronic non-communicable diseases and coverage of primary care: analysis of indicators

Mortalidad prematura por enfermedades crónicas no transmisibles y cobertura de atención: análisis de los indicadores

\section{Samara Eliane Rabelo Suplici ${ }^{I}$, Sabrina da Silva de Souza ${ }^{I I}$, Katheri Maris Zamprogna ${ }^{\text {III }}$ Ana Cláudia da Cunha ${ }^{\mathrm{Iv}}$, Dione Lúcia Prim Laurindov}

Resumo: Objetivo: avaliar a taxa de mortalidade prematura por doenças crônicas não transmissíveis (DCNT) e sua associação com a cobertura populacional pelas equipes de Atenção Básica nos municípios de Santa Catarina. Método: estudo transversal que utilizou dados secundários do banco de dados oficiais do Estado. Os indicadores mortalidade prematura por DCNT e cobertura populacional pelas equipes de Atenção Básica nos anos 2017 e 2018, foram coletados em 2019. Os dados foram analisados no Statistical Package for the Social Sciences versão 25.0, para estatística descritiva e apresentados por média e desvio padrão. Utilizou-se teste de correlação de Spearman para as variáveis em estudo. Resultados: houve aumento nas taxas de mortalidade prematura por DCNT e redução na cobertura populacional pelas equipes de Atenção Básica, sem associação significativa entre esses indicadores. Conclusão: o aumento da mortalidade prematura por DCNT e o não cumprimento das metas pactuadas requerem avaliação das ações realizadas na Atenção Básica.

Descritores: Mortalidade Prematura; Doenças não Transmissíveis; Atenção Primária à Saúde; Saúde da Família; Promoção da Saúde

Abstract: Objective: to evaluate the rate of premature mortality due to chronic noncommunicable diseases (CNCD) and its association with population Coverage of Primary Care teams in Santa Catarina municipalities.

\footnotetext{
${ }^{\text {I }}$ Enfermeira, Doutora em Enfermagem pela Universidade Federal de Santa Catarina (UFSC), membro do grupo de pesquisas NUCRON/UFSC, Florianópolis, Santa Catarina, Brasil. E-mail: samara.suplici@gmail.com, Orcid http://orcidorg/0000-0002-0334-7195

II Enfermeira, Doutora em Enfermagem pela Universidade Federal de Santa Catarina (UFSC), Hospital Universitário/UFSC e Secretaria Municipal de São José, São José, Santa Catarina, Brasil. E-mail: enfermeirasabrina@gmail.com, Orcid: http://orcidorg/0000-0001-9046-6434

III Enfermeira, Doutora em Enfermagem pela Universidade Federal de Santa Catarina (UFSC), Florianópolis, Santa Catarina, Brasil. E-mail: katherizamprogna@gmail.com, Orcid: http://orcidorg/0000-0002-5987-1025

IV Enfermeira. Especialista em Enfermagem na Saúde da Família (UFSC), Secretaria Municipal de São José, São José, Santa Catarina, Brasil. Email: anacunha1905@gmail.com, Orcid http://orcidorg/0000-0003-2292-3917

v Enfermeira. Especialista em Enfermagem na Saúde da Família (UFSC), Secretaria Municipal de São José, São José, Santa Catarina, Brasil. Email: dione.prim@gmail.com, Orcid http://orcidorg/0000-001-6233-2527
} 
Method: cross-sectional study that used secondary data from the official state database. The indicators premature Mortality due to CNCD and population Coverage of Primary Care teams in the years 2017 and 2018 were collected in 2019. The data were analyzed in the Statistical Package for the Social Sciences version 25.0, for descriptive statistics and presented by mean and standard deviation. Spearman correlation test was used for the variables under study. Results: there was an increase in the rates of premature Mortality due to CNCD and a reduction in population Coverage of Primary Care teams, with no significant association between these indicators. Conclusion: the increase in premature mortality due to $\mathrm{CNCD}$ and the non-compliance with the agreed goals require evaluation of the actions performed in Primary Care.

Descriptors: Mortality, Premature; Noncommunicable Diseases; Primary Health Care; Family health; Health Promotion

Resumen: Objetivo: evaluar la tasa de mortalidad prematura por enfermedades crónicas no transmisibles (ECNT) y su asociación con la cobertura poblacional por parte de los equipos de atención primaria en los municipios de Santa Catarina. Método: estudio transversal que utilizó datos secundarios de la base de datos oficial del estado. Los indicadores Mortalidad prematura por ECNT y cobertura poblacional por parte de los equipos de atención primaria en los años 2017 y 2018 se recogieron en 2019. Los datos fueron analizados en el Statistical Package for the Social Sciences versión 25.0, para estadística descriptiva y presentados por media y desviación estándar. La prueba de correlación de Spearman se utilizó para las variables en estudio. Resultados: hubo aumento en las tasas de mortalidad prematura debido a la ECNT y reducción en la cobertura poblacional por parte de los equipos de atención primaria, sin una asociación significativa entre estos indicadores. Conclusión: el aumento de la Mortalidad prematura por ECNT y el incumplimiento de las metas acordadas requiere evaluación de las acciones realizadas en Atención Primaria.

Descriptores: Mortalidad Prematura; Enfermedades no Transmisibles; Atención Primaria de Salud; Salud de la Familia; Promoción de la Salud

\section{Introdução}

As Doenças Crônicas Não Transmissíveis (DCNT) constituem um problema de magnitude mundial sendo responsáveis por uma diminuição da qualidade de vida, impactos econômicos negativos para famílias, comunidades e sociedade e ainda, por um elevado número de mortes. ${ }^{1}$ De acordo com a Organização Mundial da Saúde (OMS), as DCNT levam a óbito cerca de 38 milhões de indivíduos a cada ano, sendo responsáveis por $70 \%$ das mortes no mundo. ${ }^{2}$ No Brasil, elas correspondem a $75 \%$ das causas de mortes. ${ }^{1,3}$

Nos países em desenvolvimento cerca de um terço dos óbitos por DCNT afetam pessoas com menos de 60 anos de idade, enquanto nos países desenvolvidos a mortalidade prematura 
(faixa etária de 30 a 69 anos) corresponde a menos de $13 \%$ dos casos. ${ }^{4}$ A taxa de mortalidade prematura pelo conjunto das quatro principais DCNT (doenças do aparelho circulatório, câncer, diabetes e doenças respiratórias crônicas) é um indicador de saúde utilizado em todo o mundo para acompanhar uma das metas propostas para a área da saúde nos Objetivos do Desenvolvimento Sustentável (ODS). No Brasil, este indicador contribui para o monitoramento da mortalidade por estas causas que são responsáveis pelo maior número de óbitos em todo o país. Além disso, é um importante parâmetro para o planejamento e pactuação dos serviços de saúde, em todos os níveis de atenção, voltados às pessoas com doenças crônicas. Seu uso para o monitoramento da qualidade da atenção à saúde e bem-estar da população de 30 a 69 anos devese ao reconhecimento da evitabilidade da morte até esta idade. ${ }^{5}$

Com o objetivo de reduzir e prevenir os riscos e agravos à saúde da população, com foco na prevenção de DCNT, a taxa de mortalidade prematura é um dos indicadores da pactuação interfederativa, relacionados a prioridades nacionais em saúde. ${ }^{5}$ Esse indicador faz parte do Plano de Ações Estratégicas para o Enfrentamento das DCNT no Brasil para o período de 2011 a $2022^{6}$ que está alinhado ao Plano Global de Ações para a Prevenção e Controle das DCNT 2013$2020,{ }^{7}$ da OMS. No âmbito global, o plano prevễ redução de $25 \%$ na mortalidade prematura por estas causas até 2025. O Brasil estabeleceu a meta de reduzir a mortalidade prematura em $2 \%$ ao ano. $^{6}$

A literatura aponta que um dos fatores responsáveis por uma redução de aproximadamente $20 \%$ nas taxas de mortalidade por DCNT é expansão da Atenção Básica. Na última década, uma maior cobertura nesta área contribuiu para um avanço na saúde dos brasileiros. $^{8}$ A cobertura populacional pelas equipes de Atenção Básica tem sido considerada ordenadora do cuidado e eixo estruturante de programas e projetos, consolidando-se num indicador fundamental. ${ }^{5}$ 
\begin{tabular}{ll|l} 
Mortalidade prematura por doenças crônicas não transmissíveis e cobertura da atenção... & 4
\end{tabular}

A pactuação tem a finalidade de reforçar as responsabilidades de cada gestor com as necessidades de saúde da população no território, além de fortalecer a integração dos instrumentos de planejamento no Sistema Único de Saúde (SUS)..$^{8-9}$ Nesse sentido, os indicadores de saúde são instrumentos para a avaliação do desempenho das ações dos serviços. As informações determinadas pelos indicadores proporcionam a fundamentação indispensável à avaliação das ações realizadas pelo sistema de saúde. ${ }^{9}$

Existem evidências de que uma atuação adequada da Atenção Básica reduz os óbitos por DCNT..$^{10-11}$ No entanto, permanece uma lacuna no que tange a possível associação entre a mortalidade prematura por DCNT e a cobertura populacional pelas equipes de Atenção Básica. Tem-se como questão de pesquisa: Existe associação entre a taxa de mortalidade prematura por DCNT com a cobertura populacional pelas equipes de Atenção Básica nos municípios catarinenses? Este estudo partiu da hipótese de que uma menor taxa de mortalidade prematura por DCNT está associada à maior cobertura populacional pelas equipes de Atenção Básica. O objetivo foi avaliar a taxa de mortalidade prematura por DCNT e sua associação com a cobertura populacional pelas equipes de Atenção Básica nos municípios de Santa Catarina.

\section{Método}

Estudo do tipo ecológico transversal realizado com base em dados secundários da pactuação interfederativa 2017 - 2021. ${ }^{5}$ Os dados foram extraídos do banco de dados oficiais da Diretoria de Vigilância Epidemiológica do Estado de Santa Catarina (DIVE/SC). ${ }^{12}$ O período estudado foram os anos 2017 e 2018 sendo objeto de estudo todos os seus 295 municípios.

A variável dependente foi a taxa de mortalidade prematura por DCNT. Este indicador considera o número de óbitos prematuros em determinado ano e local registrados nos códigos CID-10: I00-I99 (doenças do aparelho circulatório); C00-C97 (neoplasias malignas); J30-J98 
exceto J36 (doenças do sistema respiratório) e E10-E14 (Diabetes Mellitus). O método de cálculo tem como numerador o número de óbitos (de 30 a 69 anos), pelas doenças acima mencionadas, registrados em determinado ano e local. O denominador é a população residente (de 30 a 69 anos) em determinado ano e local. O fator de multiplicação é 100.000. Desse modo, a unidade de medida é expressa em número de óbitos por 100.000 habitantes. É utilizado para o monitoramento do impacto das políticas públicas na prevenção e no controle dessas doenças e de seus fatores de risco. ${ }^{5}$

A variável independente foi a taxa de cobertura populacional pelas equipes de Atenção Básica. Este indicador considera o valor de 3.450 indivíduos cobertos por equipe de Saúde da Família, e 3.000 indivíduos pelas equipes de Atenção Básica parametrizadas e equipes equivalentes. O método de cálculo tem como numerador: o número de equipes de Saúde da Família x 3.450 + (número de equipes de Atenção Básica parametrizadas e equipes equivalentes) x 3.000 em determinado local e período. O denominador é a estimativa da populacional do ano anterior. O fator de multiplicação é 100, desse modo, a unidade de medida é expressa em porcentagem. Esta taxa é utilizada para o monitoramento do acesso e capacidade de resolução dos serviços de Atenção Básica, com vistas ao fortalecimento do planejamento do SUS de acordo com a pactuação interfederativa $2017-2021 .^{5}$

Os dados foram coletados em setembro de 2019 e organizados em uma planilha no Excel 2016 (Microsoft Office) com dupla digitação. As análises foram realizadas nos softwares Statistical Package for the Social Sciences (SPSS), versão 25.0 da plataforma de software estatístico potente IBM e R - um ambiente com um conjunto integrado de recursos de software para tratamento de dados, cálculos e exibição gráfica. ${ }^{13}$ As estatísticas descritivas contemplaram médias, medianas, desvios-padrão e intervalo interquartil (IQ) Q1-Q3 (25-75\%) para os indicadores do estudo. Para testar a hipótese de que os indicadores selecionados não eram correlacionados entre si foi proposta uma matriz de correlação a partir do cálculo do coeficiente 
Mortalidade prematura por doenças crônicas não transmissíveis e cobertura da atenção... |6

de correlação de postos de Spearman. Os testes levaram em consideração um $\alpha$ bidirecional de 0,05 e um intervalo de confiança (IC) de 95\%.

Foram plotados mapas coropléticos com os indicadores selecionados para avaliação e distribuição na área de estudo, no período 2017-2018. Foi utilizado o software Quantum Geographic Information Sysstem, uma multiplataforma de sistema de informação geográfica, de acesso livre, que permite visualização, edição e análise de dados georreferenciados. ${ }^{14}$

Este estudo é parte de um macroprojeto que avaliou a cobertura populacional pelas equipes de Atenção Básica nos municípios de Santa Catarina e o alcance dos indicadores de acordo com os dados da pactuação interfederativa 2017-2021. Neste macroprojeto cada indicador foi tratado como uma variável de análise. Como a pactuação interfederativa possui 23 indicadores, cada um deles foi identificado pela letra V (indicando tratar-se de uma variável) seguida de uma numeração arábica (de 1 a 23). Assim, na matriz de correlação de Spearman, apresentada no presente trabalho, a variável taxa de mortalidade prematura por DCNT será apresentada como V5 e a variável cobertura populacional pelas equipes de Atenção Básica será apresentada como V23.

Os aspectos éticos da pesquisa com seres humanos ocorreram no curso da investigação, de acordo com a resolução no 466 de 2012 do Conselho Nacional de Saúde e complementares. Este trabalho se deu a partir de fontes de dados secundários, de domínio público e, portanto, não precisou ser avaliada por comitê de ética. A pesquisa não implicou em risco para os sujeitos e seguiu os princípios éticos de respeito pela pessoa, caridade e justiça, diretrizes e padrões regulatórios.

\section{Resultados}

A taxa de mortalidade prematura pelas DCNT aumentou em Santa Catarina entre os anos de 2017 e 2018. Este indicador apresentou média de 312 em 2017 e 312,7 em 2018. A cobertura 
populacional pelas equipes de atenção básica apresentou médias menores no ano de 2018 se comparado ao ano de 2017, tendo médias de 82,8 em 2017 e 82,4 em 2018 (Tabela 1).

Tabela 1 - Taxa de mortalidade prematura por doenças crônicas não transmissíveis e cobertura populacional pelas equipes de Atenção Básica no estado de Santa Catarina nos anos de 2017 e 2018. Brasil, 2019

\begin{tabular}{|c|c|c|c|c|c|c|}
\hline Indicador & Ano & Média & Mediana & $\begin{array}{l}\text { Desvio } \\
\text { Padrão }\end{array}$ & $\begin{array}{c}\text { Percentil } \\
25\end{array}$ & $\begin{array}{c}\text { Percentil } \\
75\end{array}$ \\
\hline \multirow[t]{2}{*}{ Taxa de mortalidade prematura por DCNT* } & 2017 & 312.0 & 304.3 & 127.9 & 234.9 & 387.5 \\
\hline & 2018 & 312.7 & 305.5 & 126.7 & 231.2 & 373.3 \\
\hline \multirow[t]{2}{*}{ Cobertura populacional pelas equipes ${ }^{\dagger}$} & 2017 & 82.8 & 100.0 & 25.3 & 65.4 & 100.0 \\
\hline & 2018 & 82.4 & 100.0 & 25.5 & 66.7 & 100.0 \\
\hline
\end{tabular}

Fonte: DIVE/SC

* número de óbitos na população entre 30 e 69 anos de idade por 100.000 habitantes entre 30 e 69 anos de idade; †porcentagem de indivíduos cobertos por equipe de Saúde da Família, por equipes de Atenção Básica parametrizadas e equipes equivalentes

A matriz de correlação mostrada nas Figuras 1 e 2 apresenta os vários indicadores pactuados no Estado de Santa Catarina nos anos de 2017 e 2018. No presente estudo, foi avaliado a correlação do indicador "taxa de mortalidade prematura por DCNT" (V5) e "cobertura populacional pelas equipes de Atenção Básica” (V23). O coeficiente de correlação está na barra de cores, então quanto mais escura é a cor mais bem correlacionado é o indicador. As bolinhas que aparecem em segundo plano (mais claras) na matriz foram aquelas não significantes do ponto de vista estatístico $(\mathrm{P}>0,05)$.

Figura 1 - Matriz de correlação da taxa de mortalidade prematura por doenças crônicas não transmissíveis (V5) e cobertura populacional pelas equipes de Atenção Básica (V23) no estado de Santa Catarina no ano de 2017. Brasil, 2019 
\begin{tabular}{ll|l} 
Mortalidade prematura por doenças crônicas não transmissíveis e cobertura da atenção... & 8
\end{tabular}

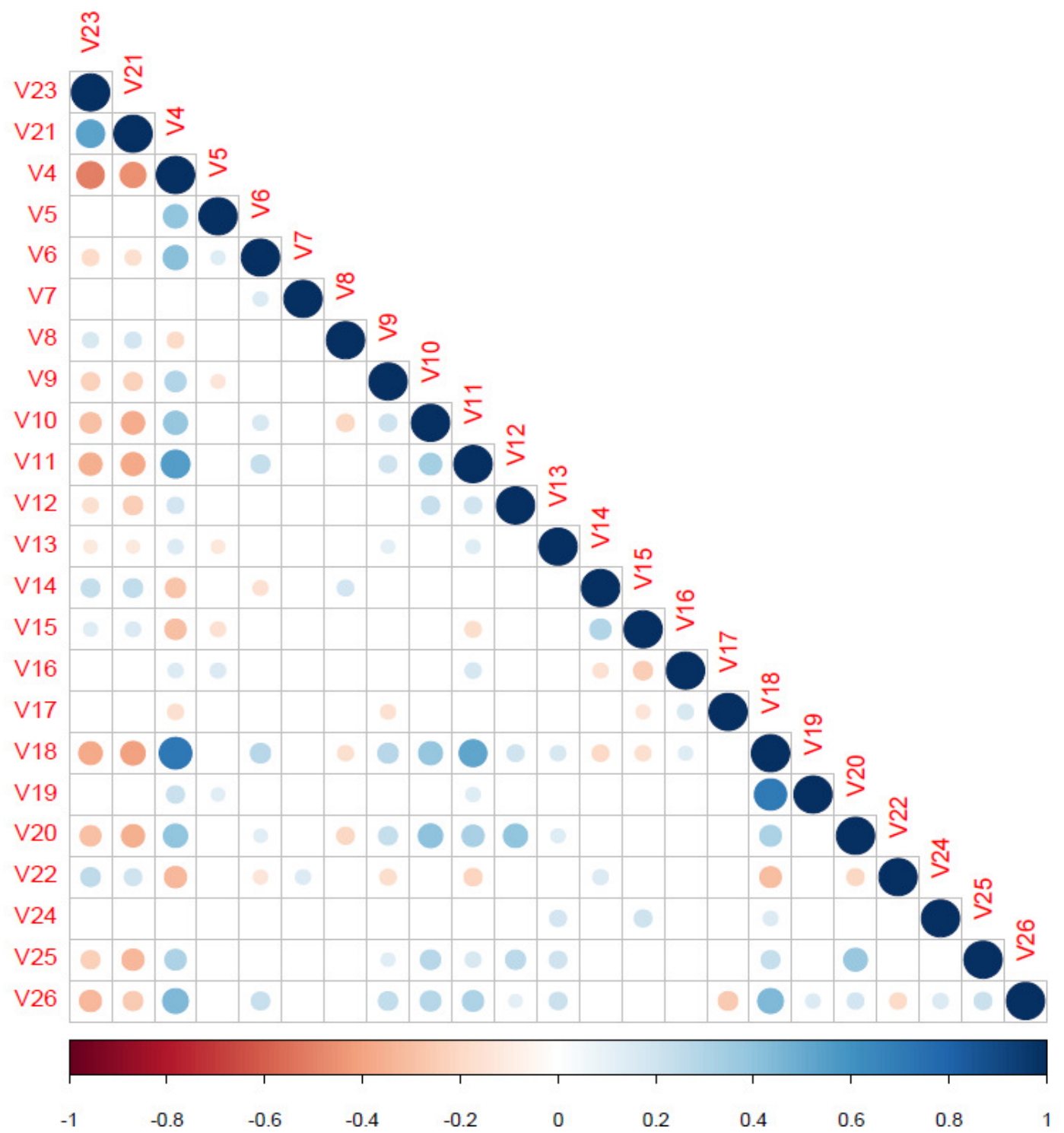

Teste de correlação de Spearman

Figura 2 - Matriz de correlação da taxa de mortalidade prematura por doenças crônicas não transmissíveis (V5) e cobertura populacional pelas equipes de Atenção Básica (V23) no estado de Santa Catarina no ano de 2018. Brasil, 2019 
9 | Suplici SER, Souza SS, Zamprogna KM, Cunha AC, Laurindo DLP

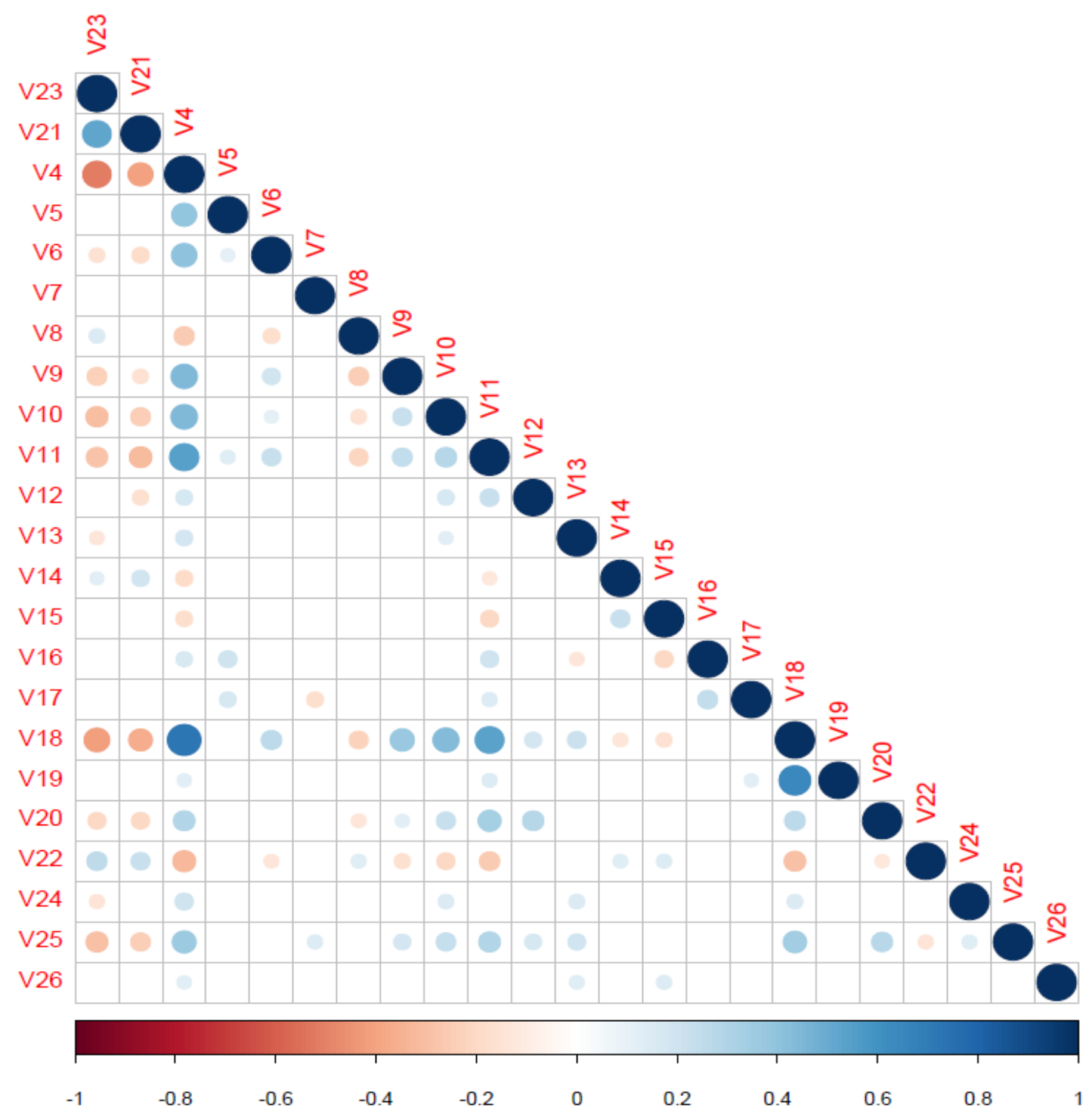

Teste de correlação de Spearman

A partir da matriz de correlação apresentada nas Figuras 1 e 2 ficou evidenciado que a taxa de mortalidade prematura por DCNT não teve correlação com a cobertura populacional pelas equipes de Atenção Básica nos anos de 2017 e 2018. Isso porque, as bolinhas aparecem em segundo plano na interseção entre V5 e V23, indicando que a correlação entre estes indicadores não apresentou significância do ponto de vista estatístico. Ainda no que se refere à taxa de mortalidade prematura por DCNT, a Figura 3 apresenta sua distribuição nos diferentes municípios de Santa Catarina, nos anos de 2017 e 2018, por meio de mapa temático.

Figura 3 - Mapa temático do indicador taxa de mortalidade prematura por doenças crônicas não transmissíveis no estado de Santa Catarina nos anos de 2017 e 2018. Brasil, 2019 

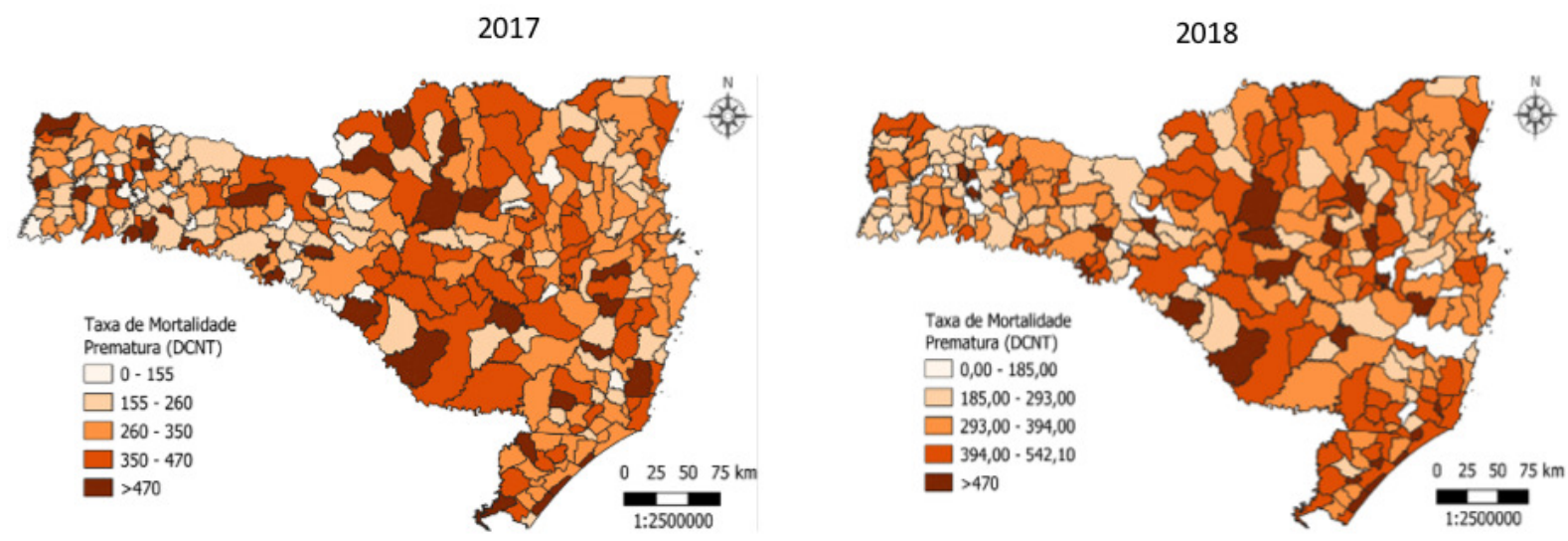

No mapa apresentado na Figura 3, quanto mais escura é a cor do município, maiores são as taxas de mortalidade prematura por DCNT. Quando comparadas as taxas de mortalidade por DCNT no período estudado, pode-se observar que em 2017, 30 municípios tiveram taxa de mortalidade por DCNT entre 0-155, 65 municípios entre 155-260, 99 municípios entre 260-350, 65 municípios entre 350-470 e 36 municípios acima de 470. Já no ano de 2018 foi observado que 25 municípios tiveram taxa de mortalidade por DCNT entre 0-155, 70 municípios entre 155-260, 97 municípios entre 260-350, 76 municípios entre 350-470 e 27 municípios acima de 470.

\section{Discussão}

O presente estudo evidenciou um aumento na taxa de mortalidade prematura por DCNT no Estado de Santa Catarina entre os anos de 2017 e 2018. Esse aumento também é observado nos municípios individualmente conforme mostrado nos mapas da Figura 3. Esse dado difere do observado em outros países e, também do Brasil como um todo, cujas pesquisas têm mostrado uma redução das taxas desse indicador, pois trata-se de doenças sensíveis às intervenções de promoção da saúde e assistência. ${ }^{10,15} \mathrm{Um}$ estudo que avaliou a série temporal da taxa de mortalidade prematura por DCNT de 2000 a 2013, no Brasil, evidenciou declínio em 2,5\% ao ano. Todas as regiões brasileiras apresentaram tendência de declínio deste indicador, sendo que as taxas do Nordeste ultrapassam as das demais regiões. ${ }^{4}$ Outro estudo que verificou a variação da 
11 Suplici SER, Souza SS, Zamprogna KM, Cunha AC, Laurindo DLP

taxa de mortalidade prematura por DCNT nas unidades federativas do Brasil e no Distrito Federal, no período de 2006 a 2014 mostrou que houve diminuição significativa deste indicador no país sendo que Santa Catarina teve redução de $2,8 \%{ }^{16}$

Neste estudo, no entanto, pode-se observar que, no período estudado, 115 municípios tiveram aumento da taxa de mortalidade prematura por DCNT, o que corresponde a $38,98 \%$ dos municípios catarinenses. Os demais reduziram ou mantiveram a mesma taxa. Portanto, apesar de uma tendência de queda deste indicador no estado, observada em anos anteriores, ainda existem flutuações. O aumento deste indicador em Santa Catarina entre os anos de 2017 e 2018, evidenciado neste estudo, ressalta a necessidade de avaliação das políticas públicas de saúde relacionadas à redução da mortalidade prematura por DCNT no estado. Ela somente será possível com intervenções de promoção da saúde e da assistência uma vez que as DCNT são as maiores causas de morte nas cinco regiões brasileiras. ${ }^{4}$

Os indicadores de pactuação, dentre os quais está a taxa de mortalidade prematura, não são simplesmente números, mas atribuições de valor a objetivos, acontecimentos ou situações, de acordo com o resultado final pretendido. Eles são essenciais nos processos de monitoramento e avaliação, pois permitem acompanhar o alcance das metas. ${ }^{5}$

A redução da probabilidade de morte prematura por DCNT em 30\% até 2030 foi um dos ODS proposto pela Assembleia das Nações em 2015, dando continuidade ao compromisso já assumido pela Assembleia Mundial de Saúde. ${ }^{4}$ No Brasil, conforme já mencionado, o objetivo é reduzir a taxa de mortalidade prematura em $2 \%$ ao ano até $2022 .{ }^{6}$ A partir desse parâmetro nacional, as metas para este indicador são estabelecidas pelos estados individualmente, de acordo com as taxas apresentadas nos anos anteriores. ${ }^{5}$

A meta pactuada pelo estado de Santa Catarina foi 283 em 2017 e 277 em $2018 .{ }^{17}$ Nesse sentido, cabe destacar que, no período estudado, Santa Catarina não atingiu as metas propostas na taxa de mortalidade prematura por DCNT. Este dado difere de outro estudo que evidenciou 
que o Brasil tem tido a capacidade de alcançar estas metas, ${ }^{4}$ reforçando a necessidade de análise crítica dos resultados obtidos e do processo de tomada de decisão, bem como de melhoria contínua dos processos organizacionais relacionados a este indicador no estado. A redução das taxas de mortalidade prematura depende da implantação de políticas públicas de saúde para prevenção e controle das doenças crônicas, bem como dos seus fatores de risco como o tabagismo, consumo de álcool, dieta não saudável e inatividade física ${ }^{18}$ sendo que para atingir esses objetivos é fundamental uma Atenção Básica forte e preparada.

Nesse sentido, este estudo evidenciou uma diminuição da cobertura populacional pelas equipes de Atenção Básica entre os anos de 2017 e 2018. Além disso, o Estado não alcançou as metas pactuadas para este indicador nos anos de 2017 e 2018 que foram 88,35\% e 88,16\%, respectivamente. ${ }^{17}$ Não há um parâmetro nacional definido para este indicador, sendo que a meta pactuada pelos estados deve considerar a realidade epidemiológica de cada ente federado.

Cabe destacar, que quanto maior a cobertura populacional pela Atenção Básica maior será o potencial de oferta das ações e serviços de saúde básicos para a população. Apesar desse indicador mensurar a carga horária de trabalho dos profissionais e não o trabalho efetivamente realizado por eles, aferir a oferta potencial dos serviços na Atenção Básica ajuda a compreender o acesso a esse nível de atenção. ${ }^{19}$ A diminuição desse indicador no estado também merece uma discussão sobre ações que possam fortalecer as equipes de Atenção Básica.

A literatura aponta que o primeiro estudo, baseado em dados nacionais, que estimou e discutiu as coberturas da Atenção Básica foi publicado em 2017 com dados de 2012. Este estudo evidenciou altas coberturas da Atenção Básica na maioria dos estados brasileiros, sendo superiores a 70\%. Este mesmo estudo destaca a importância de novos estudos que acompanhem e avaliem esse indicador. ${ }^{19}$

Foi evidenciado que a cobertura populacional pelas equipes de Atenção Básica não influenciou a taxa de mortalidade prematura por DCNT. Este dado, por sua vez, está em 
consonância com um estudo que confirmou a hipótese de que a cobertura universal é um elemento chave para alcançar objetivos globais, mas também mostrou piores indicadores de saúde (relacionados à Tuberculose) em municípios com maior cobertura da Atenção Básica. ${ }^{20}$

Pode-se inferir que tendo como diretriz a ampliação e qualificação do acesso aos serviços de saúde de qualidade, com ênfase no cuidado humanizado, na equidade e no atendimento das necessidades de saúde, uma melhor cobertura populacional pelas equipes de Atenção Básica pode trazer melhores resultados na saúde das pessoas. ${ }^{5}$ No entanto, individualmente pode não ser o suficiente para reduzir a mortalidade prematura por DCNT. Isso porque, a cobertura da Atenção Básica ordena o cuidado no sistema de saúde e favorece sua capacidade resolutiva, ${ }^{5}$ no entanto, outros aspectos também são mencionados na literatura no sentido da redução da mortalidade prematura por DCNT.

Dentre estes aspectos, vale destacar que outro estudo ${ }^{21}$ apontou uma associação entre determinantes sociais (piores condições socioeconômicas) e piores desfechos em saúde (maior susceptibilidade ao desenvolvimento das DCNT, bem como suas comorbidades e maiores taxas de mortalidade). Os determinantes sociais geram padrões de vida que causam danos que se acumulam ao longo da vida podendo levar às DCNT e à morte prematura causada por elas. ${ }^{21-24}$ Desse modo, apesar deste estudo não ter evidenciado uma relação causal entre a cobertura populacional pelas equipes de Atenção Básica e a taxa de mortalidade prematura por DCNT, ele buscou apontar a multicausalidade da morte prematura por estas condições e a importância dos estudos nesta área se dedicarem à identificação das suas variáveis preditoras. Por isso, é necessário um aprofundamento destes achados com outras pesquisas que incluam outras variáveis de análise para compreender esse fenômeno.

Dentre as limitações deste estudo, destaca-se que, apesar dos esforços no sentido da redução dos subregistros, podem ocorrer eventos não identificados na utilização de dados 
Mortalidade prematura por doenças crônicas não transmissíveis e cobertura da atenção... | 14

secundários. Essa limitação não reduz a confiabilidade do estudo, mas deve ser considerada na interpretação dos resultados.

\section{Conclusão}

Este estudo evidenciou que o estado de Santa Catarina aumentou suas taxas de mortalidade prematura por DCNT no período estudado e não atingiu as metas pactuadas para o período. A cobertura populacional pelas equipes de Atenção Básica diminuiu entre os anos de 2017 e 2018 em Santa Catarina e este fato não teve associação com o aumento da taxa de mortalidade prematura por DCNT.

Isso mostra que apesar das tendências de declínio deste indicador no Brasil e dos esforços no sentido de consolidar a Atenção Básica, o estado ainda apresenta instabilidade no controle da mortalidade prematura por DCNT. Nesse sentido, é preciso além de considerar as políticas de prevenção e controle das DCNT, considerar os fatores intervenientes na mortalidade prematura por estas condições. O modelo de atenção adotado na Atenção Básica, a avaliação das ações realizadas pelas equipes na Atenção Básica e os determinantes sociais de saúde são aspectos importantes a serem considerados na redução da taxa de mortalidade prematura por DCNT.

Por isso, as contribuições desse trabalho se dão no âmbito da gestão organizacional, pois possibilita direcionar a tomada de decisões em relação à redução das taxas de mortalidade prematura por DCNT em de Santa Catarina. Frente aos achados, recomenda-se o fortalecimento das ações de prevenção e controle da DCNT no estado e ainda, que sejam realizados futuros estudos relacionados à identificação das variáveis preditoras da mortalidade prematura por DCNT. 


\section{Referências}

1. Bernal RTI, Felisbino-Mendes MS, Carvalho QH, Pell J, Dundas R, Leyland A, et al. Indicadores de doenças crônicas não transmissíveis em mulheres com idade reprodutiva, beneficiárias e não beneficiárias do Programa Bolsa Família. Rev Bras Epidemiol. 2019;22(Suppl 2):e190012.supl.2. doi: https://doi.org/10.1590/1980-549720190012.supl.2

2. World Health Organization (WHO). Health statistics and information systems: disease burden and mortality estimates [Internet]. 2016 [cited 2020 Nov 15]. Available from: https://www.who.int/healthinfo/global_burden_disease/estimates/en/

3. Williams J, Allen L, Wickramasinghe K, Mikkelsen B, Roberts N, Townsend N. A systematic review of associations between non-communicable diseases and socioeconomic status within low- and lowermiddle-income countries. J Glob Health. 2018;8(2):020409. doi: https://doi.org/10.7189/jogh.08.020409

4. Malta DC, Andrade SSCA, Oliveira TP, Moura L, Prado RR, Souza MFM. Probabilidade de morte prematura por doenças crônicas não transmissíveis, Brasil e regiões, projeções para 2025. Rev Bras Epidemiol. 2019;22:e190030. doi: https://doi.org/10.1590/1980-549720190030

5. Conselho de Secretarias Municipais de Saúde de Santa Catarina (COSEMS/SC). Resolução CIT No. 08/2016. Pactuação interfederativa 2017/2021. Indicadores para pactuação de metas [Internet]. Florianópolis: COSEMS/SC; 2018 [acesso em 2020 nov 15]. Disponível em: https://www.cosemssc.org.br/pactuacao-de-indicadores-2018/

6. Ministério da Saúde (BR). Plano de Ações Estratégicas para o enfrentamento das Doenças Crônicas Não Transmissíveis (DCNT) no Brasil (2011-2022) [Internet]. Brasília (DF): Ministério da Saúde; 2013 [acesso em 2020 nov 15]. Disponível em: https://bvsms.saude.gov.br/bvs/publicacoes/plano_acoes_enfrent_dcnt_2011.pdf

7. World Health Organization (WHO). Global action plan for the prevention and control of noncommunicable diseases 2013-2020 [Internet]. Geneva: WHO; 2013 [cited 2020 Nov 15]. Available from: https://apps.who.int/iris/bitstream/handle/10665/94384/9789241506236_eng.pdf;jsessionid=B683C851A2F7 2909EFBC2306087E61A1? sequence $=1$

8. BRASIL. Ministério da Saúde. Portaria no 2.488, de 21 de outubro de 2011. Aprova a Política Nacional de Atenção Básica, estabelecendo a revisão de diretrizes e normas para a organização da Atenção Básica, para a Estratégia Saúde da Família (ESF) e o Programa de Agentes Comunitários de Saúde (PACS). Brasília, DF, Ministério da Saúde, 2011. Disponível em: http://bvsms.saude.gov.br/bvs/saudelegis/gm/2011/prt2488_21_10_2011.html. Acesso em: 15 nov. 2020.

9. Lima KWS, Antunes JLF, Silva ZP. Percepção dos gestores sobre o uso de indicadores nos serviços de saúde. Saúde Soc. 2015;24(1):61-71. doi: https://doi.org/10.1590/S0104-12902015000100005

10. Ministério da Saúde (BR). Saúde Brasil 2014: uma análise da situação de saúde e das causas externas 
[Internet]. Brasília (DF): Ministério da Saúde; 2015 [acesso em 2020 nov 15]. Disponível em: https://bvsms.saude.gov.br/bvs/publicacoes/saude_brasil_2014_analise_situacao.pdf

11. Ribeiro TH, Magri CL, Santos AL. Hospitalizações por Diabetes Mellitus em adultos e relação com expansão Atenção Primária no Paraná. Saúde Pesqui. 2019;12(2):323-31. doi: https://doi.org/10.17765/21769206.2019v12n2p323-331

12. Diretoria de Vigilância Epidemiológica (DIVE). Diretoria de Vigilância Epidemiológica do Estado de Santa Catarina. Indicadores - Pactuação Interfederativa 2017 a 2021 - Santa Catarina. [acesso 2020 ago 15]. Disponível em: http://200.19.223.105/cgi-bin/dh?popsc/def/popsc.def

13. The $\mathrm{R}$ Foundation. The $\mathrm{R}$ Project for Statistical Computing [Internet]. Vienna (Austria): The $\mathrm{R}$ Foundation; 2015 [cited 2019 Dec 15]. Available from: https:/www.r-project.org/

14. Open Source Geospatial Foundation (OSGeo). QGIS: a free and open source geographic information system [Internet]. Beaverton (OR): OSGeo; 2015 [cited 2019 Dec 01]. Available from: http://www.qgis.org/

15. Malta DC, Moura L, Prado RR, Escalante JC, Schmidt MI, Duncan BB. Mortalidade por doenças crônicas não transmissíveis no Brasil e suas regiões, 2000 a 2011. Epidemiol Serv Saúde [Internet]. 2014 [acesso em 2020 nov 15];23(4):599-608. Disponível em: https://www.scielo.br/pdf/ress/v23n4/2237-9622ress-23-04-00599.pdf

16. Confortin SC, Andrade SR, Draeger VM, Meneghini V, Schneider IJC, Barbosa AR. Premature mortality caused by the main chronic noncommunicable diseases in the Brazilian states. Rev Bras Enferm. 2019;72(6):1588-94. doi: https://doi.org/10.1590/0034-7167-2018-0701

17. Governo de Santa Catarina, Secretaria de Estado de Saúde. Deliberações 2018 CIB nำ 218, de 23 de agosto de 2018. Indicadores Interfederativos. Metas do rol dos indicadores interfederativos do Estado de Santa Catarina, período de para 2018, conforme Resolução da CIT no 08 de 24 de novembro de 2016, que dispõe sobre o processo de pactuação interfederativa indicadores para o período de 2017 a 2021, de acordo com as prioridades nacionais de saúde [Internet]. Florianópolis: Secretaria de Estado da Saúde; 2019 [acesso em 2020 maio 21]. Disponível em: https://www.saude.sc.gov.br/index.php/legislacao/deliberacoes-cib/deliberacoes-2018cib?limit=20\&limitstart $=100$

18. Malta DC, Oliveira TP, Santos MAS, Andrade SSCA, Silva MMA. Progress with the Strategic Action Plan for tackling chronic non-communicable diseases in Brazil, 2011-2015. Epidemiol Serv Saúde [Internet]. 2016 [cited 2020 Nov 18];25(2):373-90. Available from: https://www.scielo.br/scielo.php?pid=S2237-96222016000200373\&script=sci_abstract

19. Poças KC, Freitas LRS, Duarte EC. Censo de estrutura da Atenção Primária à Saúde no Brasil (2012): estimativas de coberturas potenciais. Epidemiol Serv Saúde. 2017;26(2):275-84. doi: https://doi.org/10.5123/s1679-49742017000200005 
20. Souza SS. Acesso e cobertura na Atenção Primária à Saúde: uma análise dos indicadores de tuberculose no Brasil. Enferm Comunitaria [Internet]. 2019 [acesso em 2020 maio 21];15:e12395. Disponível em: http://ciberindex.com/c/ec/e12395

21. Malta DC, Campos MO, Oliveira MM, Iser BPM, Berna RTI, Claro RM, et al. Prevalência de fatores de risco e proteção para doenças crônicas não transmissíveis em adultos residentes em capitais brasileiras, 2013. Epidemiol Serv Saúde. 2015;24(3):387-73. doi: https://doi.org/10.5123/S167949742015000300004

22. Malta DC, Bernal RTI, Lima MG, Araújo SSC, Silva MMA, Freitas MIF, et al. Noncommunicable diseases and the use of health services: analysis of the National Health Survey in Brazil. Rev Saúde Pública. 2017;51(Suppl 1). doi: https://doi.org/10.1590/s1518-8787.2017051000090

23. Pearce N, Ebrahim S, McKee M, Lamptey P, Barreto ML, Matheson D, et al. Global prevention and control of NCDs: limitations of the standard approach. J Public Health Policy. 2015;36(4):408-25. doi: https://doi.org/10.1057/jphp.2015.29

24. Carrapato P, Correia P, Garcia B. Determinante da saúde no Brasil: a procura da equidade na saúde. Saude Soc. 2017;26(3):676-89. doi: https://doi.org/10.1590/s0104-12902017170304

Editora Científica: Tânia Solange Bosi de Souza Magnago

Editora Associada: Carine Vendruscolo

\section{Autor correspondente}

Samara Eliane Rabelo Suplici

E-mail: samara.suplici@gmail.com

Endereço: Rodovia Jornalista Manoel de Menezes, 345, Lagoa da Conceição, Florianópolis, SC, Brasil

CEP: $88061-700$

\section{Contribuições de Autoria}

\section{1 - Samara Eliane Rabelo Suplici}

Concepção ou desenho do estudo/pesquisa, análise e/ou interpretação dos dados, revisão final com participação crítica e intelectual no manuscrito

\section{2 - Sabrina da Silva de Souza}

Concepção ou desenho do estudo/pesquisa, análise e/ou interpretação dos dados, revisão final com participação crítica e intelectual no manuscrito 


\section{3 - Katheri Maris Zamprogna}

Concepção ou desenho do estudo/pesquisa, análise e/ou interpretação dos dados, revisão final com participação crítica e intelectual no manuscrito

\section{4 - Ana Claudia da Cunha}

Concepção ou desenho do estudo/pesquisa, análise e/ou interpretação dos dados, revisão final com participação crítica e intelectual no manuscrito

\section{5 - Dione Lúcia Prim Laurindo}

Concepção ou desenho do estudo/pesquisa, análise e/ou interpretação dos dados, revisão final com participação crítica e intelectual no manuscrito

\section{Como citar este Artigo}

Suplici SER, Souza SS, Zamprogna KM, Cunha AC, Laurindo DLP. Mortalidade prematura por doenças crônicas não transmissíveis e cobertura da atenção básica: análise dos indicadores. Rev. Enferm. UFSM. 2021 [Acesso em: Anos Mês Dia]; vol.11 e24: 1-18. DOI: https://doi.org/10.5902/2179769244513 